Pacific Journal of Mathematics

ON FINITE REGULAR RINGS

E. Hartwig and Jung Duh 


\title{
ON FINITE REGULAR RINGS
}

\author{
ROBERT E. HARTWIG AND Jiang LUH
}

\begin{abstract}
Several new properties are derived for von Neumann finite rings. A comparison is made of the properties of von Neumann finite regular rings and unit regular rings, and necessary and sufficient conditions are given for a matrix ring over a regular ring to be respectively von Neumann finite or unit regular. The converse of a theorem of Henriksen is proven, namely that if $R_{n \times n}$, the $n \times n$ matrix ring over ring $R$, is unit regular, then so is the ring $R$. It is shown that if $R_{2 \times 2}$ is finite regular then $a \in R$ is unit regular if and only if there is $x \in R$ such that $R=a R+x\left(a^{o}\right)$, where $a^{o}$ denotes the right annihilator of $a$ in $R$.
\end{abstract}

1. Introduction. In [13], Henriksen posed the question whether a finite regular ring is unit regular. This was subsequently proven in part by Ehrlich [4] for a particular class of regular rings. An example of finite regular rings which are not unit regular was recently given by Bergman (1974) (see Handelman [8]). In his paper [8], Handelman showed that a regular ring $R$ is unit regular if and only if, for any finitely generated projective right $R$-modules $A, B$, and $C, A \oplus B \cong A \oplus C$ implies $B \cong C$. He also characterized unit regular rings by perspectivity on the lattices of their principal right ideals. The purpose of this paper, however, is to characterize finite regular rings and to compare their properties with unit regular rings. Some of the results of the theory of generalized inverses [1] are used to show that in a regular ring, the properties of finiteness and unit-regularity each correspond to a suitable cancellation law for principal ideals. These cancellation laws are closely related to the substitution property of Fuchs [5], and the cancellation law of Ehrlich [4]. We shall use a result by Vidav [15] to show that if the matrix ring $R_{n \times n}$ is unit regular then so is ring $R$. Let us begin by defining our concepts and by stating some useful general results. A ring $R$ is called regular if for all $a \in R$, there is a solution $a^{-} \in R$ to the equation $a x a=a$. The element $a^{-}$is called as inner or 1-inverse of a [1]. Similarly, any solution to $a x a=a, x a x=x$ is called a reflexive or $1-2$ inverse of $a$, and will be denoted by $a^{+}$. For example, $a^{-} a a^{-}$ is always such a solution. An element $a \in R$ is said to have a group inverse $a^{\sharp} \in R$ if it is a group member, i.e., a belongs to some multiplicative group of $R$. Necessary and sufficient conditions for $a^{\sharp}$ to exist are that $a^{2} R=a R, R a^{2}=R a$, or $a x a=a, x a x=x, a x=x a$, for some $x \in R$ [10]. A regular ring $R$ with unity is called unit regular 
[3] if every $\alpha \in R$ has a unit inner inverse $\alpha^{-} \in R . \quad R$ is called von Neumann or Dedekind finite, finite for short, if it contains a unity 1 and $a b=1 \Rightarrow b a=1$. It is easily seen that unit-regular rings are finite. An idempotent $e^{2}=e \in R$ is said to be finite if $e R e$ is finite. The ring of $n \times n$ matrices over $R$ will be denoted by $R_{n \times n}$, while $R^{n}$ denotes the free module of $n \times 1$ columns over $R$. Isomorphisms will be denoted by $\cong$, similarity by $\approx$, and internal and external direct sums by + and $\oplus$ respectively. As usual, the right and left annihilator of $a$ will be denoted by $a^{0}=\{x \in R$; $a x=0\}$ and ${ }^{\circ} a=$ $\{x \in R ; x a=0\}$ respectively, while the Jacobson radical is given by $\mathscr{J}(R)$. Throughout this paper all rings are assumed to have unity 1 .

For two idempotents $e, f \in R, e \sim f$ means that $e=p \hat{p}, f=\hat{p} p$ for some $p \in e R f$ and $\hat{p} \in f R e$, while $e \leqq f$ denotes the well-known [14] ordering for compatible idempotents defined by $e=e f=f e$, or equivalently by $e R e \subseteq f R f$.

We shall make continued use of the following facts which hold for idempotents $e$ and $f$ in any ring $R$ with unity [14].

(i ) $e \sim f \Longleftrightarrow e R \cong f R$ as right $R$-modules $\Longleftrightarrow R e \cong R f$ as left $R$-modules.

(ii) $e \approx f \Longleftrightarrow e \sim f$ and $1-e \sim 1-f$.

(iii) $e R=f R \Longrightarrow e \approx f$.

(iv) $e=p f q, p, q$ invertible $\Longrightarrow e \approx f$.

In addition we shall use the result that

LeMma 1. If $R$ is a ring, and $A, B, C, D$ are matrices in $R_{n \times n}$, then the following are equivalent in pairs.

(i ) $A R_{n \times n} \oplus B R_{n \times n} \cong C R_{n \times n} \oplus D R_{n \times n}$ as $R_{n \times n}$-modules.

(ii) $A R^{n} \oplus B R^{n} \cong C R^{n} \oplus D R^{n}$ as $R$-modules.

(i) $A R_{n \times n}+B R_{n \times n} \cong C R_{n \times n}+D R_{n \times n}$ as $R_{n \times n}$-modules.

(ii) $A R^{n}+B R^{n} \cong C R^{n}+D R^{n}$ as $R$-modules.

Indeed, if $\phi\left[\begin{array}{l}A X \\ B Y\end{array}\right]=\phi\left[\begin{array}{c}A \\ 0\end{array}\right] X+\phi\left[\begin{array}{l}0 \\ B\end{array}\right] Y$ is the given isomorphism in $(\alpha i)$ then the mapping $\chi$, given by

$$
\chi\left[\begin{array}{l}
A \boldsymbol{x} \\
B \boldsymbol{y}
\end{array}\right]=\phi\left[\begin{array}{c}
A \\
0
\end{array}\right] \boldsymbol{x}+\phi\left[\begin{array}{l}
0 \\
B
\end{array}\right] \boldsymbol{y}
$$

will be a desired isomorphism for ( $\alpha$ ii), while conversely if $\chi\left[\begin{array}{l}A \boldsymbol{x} \\ B \boldsymbol{y}\end{array}\right]=$ $\chi\left[\begin{array}{c}A \\ 0\end{array}\right] \boldsymbol{x}+\chi\left[\begin{array}{l}0 \\ B\end{array}\right] \boldsymbol{y}$ is the isomorphism given by $(\alpha i i)$ then the map $\phi$ defined by 


$$
\phi\left[\begin{array}{l}
A\left[\boldsymbol{x}_{1}, \cdots, \boldsymbol{x}_{n}\right] \\
B\left[\boldsymbol{y}_{1}, \cdots, \boldsymbol{y}_{n}\right]
\end{array}\right]=\left[\chi\left[\begin{array}{l}
A \boldsymbol{x}_{1} \\
B \boldsymbol{y}_{1}
\end{array}\right], \cdots, \chi\left[\begin{array}{l}
A \boldsymbol{x}_{n} \\
B \boldsymbol{y}_{n}
\end{array}\right]\right]
$$

is one of the desired $R_{n \times n}$ isomorphisms for $(\alpha i)$. Similar maps can be defined for case $(\beta)$. In particular, if $E$ and $F$ are idempotents in $R_{n \times n}$, then

$$
E \sim F \Longleftarrow E R_{n \times n} \cong F R_{n \times n} \Longleftrightarrow E R^{n} \cong F R^{n} .
$$

In addition, if for example, $E=\left[\begin{array}{ll}e_{1} & 0 \\ 0 & e_{2}\end{array}\right]$ and $F=\left[\begin{array}{ll}f_{1} & 0 \\ 0 & f_{2}\end{array}\right]$ then

$$
E \sim F \Longleftrightarrow\left[\begin{array}{l}
e_{1} R \\
e_{2} R
\end{array}\right] \cong\left[\begin{array}{l}
f_{1} R \\
f_{2} R
\end{array}\right] \Longleftrightarrow e_{1} R \oplus e_{2} R \cong f_{1} R \oplus f_{2} R .
$$

The dual result for left modules is obvious. Lastly, we note that in any ring $R$,

$$
a R=b R \Longrightarrow R a \cong R b .
$$

Indeed, if $a x=b$ and $a=b y$, then the mapping $r a \mapsto r a x$ is a desired isomorphism. Let us now turn to some useful results concerning finite rings.

\section{Finite rings.}

THEOREM 1. Let $R$ be a ring with unity 1 and suppose that $e$ and $f$ are arbitrary idempotents in $R$. The following are equivalent.

(i) $R$ is finite

(ii) $e R \subseteq f R, e \sim f \Longrightarrow e R=f R$.

(iii) $R e \subseteq R f, e \sim f \Longrightarrow R e=R f$.

(iv) $e \sim 1 \Longrightarrow e=1$.

Proof. (i) $\Rightarrow$ (ii). Let $e R \subseteq f R$ and $e \sim f . \quad$ Then $e=f e$ and $e=$ $p \hat{p}, f=\hat{p} p$ for some $p \in e R f, \hat{p} \in f R e$.

Consider now

$$
\begin{aligned}
x & =\hat{p}+(1-f)(1-e)=\hat{p}+1-f, y=p+(1-e)(1-f) \\
& =p+1-e-f+e f .
\end{aligned}
$$

Then $x y=1$ and hence, since $R$ is finite, $y=x^{-1}$.

But now $f y=f p+f-f e-f+f e f=f p-e+e f \in f e R f+$ $e R=e R f+e R \subseteq e R$. Thus $f \in e R y^{-1}=e R$ and so $f R=e R$. We may again replace $e \sim f$ by either $e R \cong f R$ or $R e \cong R f$.

(ii) $\Rightarrow$ (iv). Let $e \sim 1$ and $e R \cong R . \quad$ By (ii), $e R=R$, which implies 
that $1=e x$ for some $x$, or $e=e x=1$.

(iv) $\Rightarrow$ (i). Let $a b=1$ and set $e=b a=e^{2}$. Then $e \sim 1$, because $e=p \hat{p}, 1=\hat{p} p$ where $p=b$ and $\hat{p}=a$. In fact, $p \in e R 1=b a R$ as $p=b a b=b$ and $\hat{p} \in 1 R e=R b a$ as $\hat{p}=a b a=a$. Hence by (iii) $e=1$ and so $b a=1$ as desired. Part (iii) follows by left-right symmetry.

REMaRKs 1. The last part should be compared with the result of Vidav [15], and Fuchs [5], which states that a regular ring is unit regular if and only if $e \sim f \Rightarrow e \approx f$.

2. The second part is best possible in that neither $e$ nor $f$ may fail to be idempotent. Indeed, if $R$ is finite then $a R \cong R \nRightarrow a R=R$, as seen from the example of the ring of integers with $a=2$. On the other hand, a ring $R$ with the property that $a R \cong R \Rightarrow a R=R$ must be finite, yet need not be regular as seen from the following counterexample.

ExAmple 1. Let $R_{1}=\left[\begin{array}{ll}R & R \\ 0 & R\end{array}\right]$, where $R=R$ is the real field. Then clearly $R_{1}$ is not regular since $\left[\begin{array}{ll}0 & 1 \\ 0 & 0\end{array}\right]\left[\begin{array}{ll}a & b \\ 0 & c\end{array}\right]\left[\begin{array}{ll}0 & 1 \\ 0 & 0\end{array}\right]=\left[\begin{array}{ll}0 & 0 \\ 0 & 0\end{array}\right]$. Suppose now that $\left[\begin{array}{ll}a & b \\ 0 & c\end{array}\right] R_{1} \cong R_{1}$. We claim that $\left[\begin{array}{ll}a & b \\ 0 & c\end{array}\right] R_{1}=R_{1}$. Let $\phi:\left[\begin{array}{ll}a & b \\ 0 & c\end{array}\right] R_{1} \rightarrow$ $R_{1}$ denote the given isomorphism such that $\left[\begin{array}{ll}1 & 0 \\ 0 & 1\end{array}\right]=\phi\left(\left[\begin{array}{ll}a & b \\ 0 & c\end{array}\right] X\right)=$ $\phi\left[\begin{array}{ll}a^{\prime} & b^{\prime} \\ 0 & c^{\prime}\end{array}\right]$. Now $a^{\prime} \neq 0$ since $a^{\prime}=0$ would imply that

$$
\phi\left(\left[\begin{array}{ll}
0 & b^{\prime} \\
0 & c^{\prime}
\end{array}\right]\left[\begin{array}{ll}
1 & 0 \\
0 & 0
\end{array}\right]\right)=\phi\left[\begin{array}{ll}
0 & 0 \\
0 & 0
\end{array}\right]=0,
$$

while $\phi\left(\left[\begin{array}{ll}0 & b^{\prime} \\ 0 & c^{\prime}\end{array}\right]\right)\left[\begin{array}{ll}1 & 0 \\ 0 & 0\end{array}\right]=\left[\begin{array}{ll}1 & 0 \\ 0 & 1\end{array}\right]\left[\begin{array}{ll}1 & 0 \\ 0 & 1\end{array}\right]=\left[\begin{array}{ll}1 & 0 \\ 0 & 0\end{array}\right]$. Next we claim that $c^{\prime} \neq 0$. For if $c^{\prime}=0$, then $\phi\left(\left[\begin{array}{cc}a^{\prime} & b^{\prime} \\ 0 & 0\end{array}\right]\left[\begin{array}{rr}0 & b^{\prime} \\ 0 & -a^{\prime}\end{array}\right]\right)=\phi\left[\begin{array}{ll}0 & 0 \\ 0 & 0\end{array}\right]=0$, while $\phi\left(\left[\begin{array}{ll}a^{\prime} & b^{\prime} \\ 0 & c^{\prime}\end{array}\right]\right)\left[\begin{array}{rr}0 & b^{\prime} \\ 0 & -a^{\prime}\end{array}\right]=\left[\begin{array}{rr}0 & b^{\prime} \\ 0 & -a^{\prime}\end{array}\right]$, which implies that $\alpha^{\prime}=0$, a contradiction. Thus $a^{\prime} \neq 0 \neq c^{\prime}$ and $\left[\begin{array}{cc}a^{\prime} & b^{\prime} \\ 0 & c^{\prime}\end{array}\right]$ is invertible. Hence

$$
R_{1}=\left[\begin{array}{ll}
a^{\prime} & b^{\prime} \\
0 & c^{\prime}
\end{array}\right] R_{1} \subseteq\left[\begin{array}{ll}
a & b \\
0 & c
\end{array}\right] R_{1} \leqq R_{1}
$$

as desired.

COROLLARY 1. If $R$ is finite ring and if $e^{2}=e \in R$, then the corner ring eRe is also finite. 
Proof. Suppose that $(e a e)(e b e)=e$. Then $e R=e a e R$ and $R e=$ Rebe. Hence $R e a e=R e b e(e a e)$ and thus by (1.4) $e R=e a e R \cong e b e(e a e) R \cong$ $e R$, ensuring by Theorem 1 , that $e R=(e b e) e a e R$. This implies that $e=e b e(e a e)$, as desired. We may consequently order the compatible idempotents with the usual partial ordering relation " $\leqq$ ". The following corollary is equivalent to the one above.

COROLlaRY 2. If $R$ is a finite ring and $B$ is a subring with unity, then $B$ is finite.

Proof. Let $R$ be a finite ring and let $B$ be a subring of $R$ with unity $e$. Suppose that $x, y \in B$ and $x y=e$. If $f=y x=f^{2}$, then $(f x f)(f y f)=f$ and thus since $f R f$ is finite by Corollary 1 , it follows that $y^{2} x^{2}=f y f(f x f)=y x$. On premultiplication by $x$ and postmultiplication by $y$ this yields $y x=e y x e=e$. It is obvious that Corollary 2 implies Corollary 1. It should be noted here, that the latter result can also be proven directly, as pointed out to us by the referee.

CoRollary 3. If $R$ is a finite ring and $e^{2}=e \in R$, then the centralizer $C_{e}=\{x \in R \mid x e=e x\}$ is finite.

CoROllaRY 4. If $R$ is a finite ring and $e^{2}=e \in R$, then eae is $a$ unit in eRe if and only if eaeR $=e R$.

Proof. If eaeebe $=e$ then clearly $e R=e a e R$. Conversely, if $e R=e a e R$, then for some $x \in R$, eaex $=e=(e a e) e x e$. Since $e R e$ is finite, it follows that $e=e x e(e a e)$ implying that eae is a unit in $e R e$.

We remark that this also follows from the fact that the maximal subgroup $H_{e}$ containing $e$ is the group of units in $e R e$ and that $H_{e}=\{x \in R ; x R=e R, R x=R e\}$. Finiteness shows that $e a e R=e R \Leftrightarrow$ $R e=$ Reae.

We next obtain as a corollary the result by Kaplansky [14] p. 11 , which says that an idempotent $e$ finite if it is not equivalent to a smaller idempotent.

Corollary 5. Let $R$ be a ring and and let $e, f$ be idempotents in $R$ such that $f R f$ is finite. Then

$$
e R e \subseteq f R f, e \sim f \Longrightarrow e=f .
$$

Proof. Since $e=e f=f e, e R f=f(e R f) \subseteq f R f$. Now $e R f$ and $f R f$ are isomorphic as right $f R f$-modules, since the map erf $\mapsto \hat{p} e r f$, 
with $e=p \hat{p}, f=\hat{p} p, p \in e R f=e(f R f) f, \hat{p} \in f R e=f(f R f) e$, is an example of such an isomorphism. Hence by Theorem $1, e R f=f R f$, which ensures that $f=e x f$ for some $x$ or $e=e f=f$ as desired.

We remark that if $R$ is a unit regular ring then $e R e \cong R \Rightarrow$ $e R e=R$, as seen from the example where $R=\amalg_{i=1}^{\infty} R_{i}, R_{i}=D$ (a division ring) and $e=(0,1,1, \cdots)$. The following is a partial converse to Corollary 1.

Proposition 0 (Savage). If $R$ is a ring with unity 1 such that $f R f$ is finite for every idempotent element $f \neq 1$, then $R$ is finite.

Proof. Replace $B$ by $R$ and $e$ by 1 in the proof of Corollary 2 .

Proposition 1. Suppose $R$ is a ring with unity 1 and let $\mathscr{J}(R)$ denote its Jacobson radical. Then $R$ is finite exactly when $R / \mathscr{J}(R)$ is finite.

Proof. $\Rightarrow$ : Suppose we denote $R / \mathscr{J}(R)$ by $\bar{R}$ and the elements from $\bar{R}$ by $\bar{a}, \bar{b}$ etc. Let $\bar{a} \bar{b}=\overline{1}$. Then $1-a b \in \mathscr{J}(R)$, implying that $1-(1-a b) 1=a b$ is a unit. That is $a b c=1=c a b$ for some $c$. Since $R$ is finite it follows that $b c a=1$ and so $(\bar{b} \bar{c}) \bar{a}=1$. Hence $\bar{a}$ has left and right inverses and thus is a unit with $(\bar{a})^{-1}=\bar{b}$.

$=$ : Conversely, suppose $\bar{R}$ is finite and that $a b=1$. Then $\bar{a} \bar{b}=\overline{1} \Rightarrow \bar{b} \bar{a}=\overline{1} \Rightarrow 1-b a \in \mathscr{J}(R)$. And so $1-(1-b a) 1=b a$ is a unit in $R$ implying that $b a c=1=c b a$ for some $c \in R$. Hence $a$ has left and right inverses and thus is a unit with inverse $b=a^{-1}$. This result should be contrasted with the fact that $R / \mathscr{J}(R)$ may be unit regular without $R$ being regular (cf. [5], Lemma). Indeed, if $R$ is any nonsemisimple Artinian ring, then $R$ cannot be regular while $R / \mathscr{J}(R)$ is semisimple Artinian and hence unit regular.

Finiteness is closely related to the existence of group and Drazin inverses of the elements in $R$ [2], [10]. We recall that the left (right) index $l(a)(r(a))$ of $a \in R$ is the smallest value of $p(q)$ for which $a^{p+1} R=$ $a^{p} R\left(R \alpha^{q+1}=R a^{q}\right)$, and that if both are finite, they have to be equal, [2]. This common value is called the index $i(a)$ of $a$. A ring is called strongly $\pi$-regular if every $\alpha$ in $R$ possesses a Drazin inverse. Now $a$ has a group inverse $a^{\sharp}$ in $R$ exactly when $i(a)=0$ or 1 that is exactly when $a^{2} R=a R$ and $R a^{2}=R a$ or when $a x a=a, x a x=x$ and $a x=x a$ for some $x \in R$. The following is a generalization of the concept of finiteness.

We say that a ring $R$ satisfies property $(k, l)$ where $k$ and $l$ are nonnegative integers if, for $a \in R, a^{k+1} R=a^{k} R \Rightarrow R a^{l+1}=R a^{l}$. Clearly 
all strongly $\pi$-regular rings satisfy this property [2].

Proposition 2. Let $R$ be a ring with unity 1. Suppose $R$ satisfies property $(k, l)$ for some nonnegative integers $k$ and $l$. Then $R$ is finite.

Proof. This follows immediately from the fact that if $a b=1$ then $i(a)=r(a)=l(a)=0$.

It is unknown if the converse is true in general, but we shall see shortly that this is the case for subrings of finite regular rings. Presently we do have the following result dealing with the ring $\left[\begin{array}{ll}R & R \\ 0 & R\end{array}\right]$. This ring will be used repeatedly as a source of examples and counter examples.

Proposition 3. Let $R$ be a ring with unity and let $R_{1}=\left[\begin{array}{ll}R & R \\ 0 & R\end{array}\right]$. Then

(i) $R$ is finite if and only if $R_{1}$ is finite

(ii) if $R$ has property $(1,1)$ then so does $R_{1}$, in which case both $R$ and $R_{1}$ are finite.

Proof. Since part (i) is easily established we turn to (ii). Suppose that $M=\left[\begin{array}{ll}u & v \\ 0 & w\end{array}\right], X=\left[\begin{array}{ll}a & c \\ 0 & d\end{array}\right]$ and $M^{2} X=M$. Equating entries shows that

$$
\begin{array}{ll}
\text { (i) } & u^{2} a=u \\
\text { (ii) } & w^{2} d=w \\
\text { (iii) } & u^{2} c+(u v+v w) d=v .
\end{array}
$$

Since $R$ has property $(1,1), u^{2} R=u R \Rightarrow R u^{2}=R u$ and thus $u^{\sharp}$ exists. Similarly $w^{\sharp} \in R$. Hence

$$
u u^{\sharp} u^{2}=u^{2} \text { and } \quad w d=w^{\sharp} w^{2} d=w^{\sharp} w .
$$

Then multiplying (2.3)-iii through on the left by $u u^{\sharp}$ yields

$$
u^{2} c+u v d+u u^{\sharp} v w^{\sharp} w=u u^{\sharp} v .
$$

Substracting this from (2.3)-iii gives

$$
\left(1-u u^{\sharp}\right) v\left(1-w w^{\sharp}\right)=0 .
$$

This, however, is exactly the consistency condition needed for $M$ to have a group inverse: $M^{\sharp}=\left[\begin{array}{cc}u^{\sharp} & c \\ 0 & w^{\sharp}\end{array}\right]$, where $c=-u^{\sharp} v w^{\sharp}+\left(1-u u^{\sharp}\right) v w^{\sharp 2}+$ $u^{\sharp 2} v\left(1-w w^{\sharp}\right)$, satisfying $M M^{\sharp} M=M, M^{\sharp} M M^{\sharp}=M^{\sharp}$ and $M M^{\sharp}=M^{\sharp} M$. 
It is clear that $M^{*} \in R_{1}$. For semi-simple rings the first part of this proposition may be proven with aid of Proposition 1. It is not known if the $(k, l)$ property is inherited by $R_{2 \times 2}$.

3. Finite regular rings. Let us first give a couple of preliminary observations for regular rings.

Proposition 4. Let $R$ be a regular ring and let $a, b \in R$.

$$
\text { (i) } R a \cong R b \Longrightarrow a R \cong b R
$$

(ii) If $a^{-}$and $a^{=}$are two inner inverses of $a$, then $a \alpha^{-} \sim \alpha \alpha^{=}, \alpha^{-} \alpha \sim \alpha^{=} \alpha, 1-\alpha \alpha^{-} \sim 1-\alpha \alpha^{=}$, and $1-\alpha^{-} \alpha \sim 1-\alpha^{=} \alpha$.

Proof. (i) Let $R a \cong R b$. Then $R a^{+} a \cong R b^{+} b$ and so $a^{+} a \sim b^{+} b$. Hence $a^{+} a=p \hat{p}, b^{+} b=\hat{p} p$ for some $p \in a^{+} a R b^{+} b=a^{+} R b$, and $\hat{p} \in$ $b^{+} b R a^{+} a=b^{+} R a$. If we set $q=a p b^{+}$and $\hat{q}=b \hat{p} a^{+}$, then clearly $q \hat{q}=a a^{+}, \hat{q} q=b b^{+}$, while $q \in a R b^{+}$and $\hat{q} \in b R a^{+}$. Thus $a R=a a^{+} R \cong$ $b b^{+} R=b R$. The converse follows by symmetry.

(ii) Clearly $a \alpha^{-} R=\alpha \alpha^{=} R, R \alpha^{-} \alpha=R \alpha^{=} \alpha$ and $a^{0}=\left(1-a^{-} a\right) R=$ $\left(1-a^{=} a\right) R,{ }^{\circ} a=R\left(1-a a^{-}\right)=R\left(1-a a^{=}\right)$.

If $R S(A)$ and $C S(A)$ denote the row-space and column space of a matrix $A$ respectively [1], then we have:

COROLlaRY 6. For rectangular matrixes over a regular ring $R$,

$$
R S(A) \cong R S(B) \Longleftrightarrow C S(A) \cong C S(B) \text {. }
$$

For future reference we add the following.

Proposition 5. In a regular ring $R$,

$$
\begin{aligned}
& \text { (i ) } \quad a b R=a R \Longleftrightarrow{ }^{\circ} b \cap R a=(0) \Longleftrightarrow b R+\left(1-a^{-} a\right) R=R \\
& \text { (ii) } \quad R a b=R b \Longleftrightarrow a^{o} \cap b R=(0) \Longleftrightarrow R a+R\left(1-b b^{-}\right)=R .
\end{aligned}
$$

Proof. First we recall that $a b R=a R \Rightarrow^{\circ}(a b)={ }^{\circ} a$. Now let $x \in{ }^{\circ} b \cap R a$, that is $x b=0$ and $x=y a$ for some $y \in R$. Thus if $a b R=$ $a R$, then $0=x b=y a b \Rightarrow 0=y a=x$. If we now write ${ }^{\circ} b \cap R a=$ ${ }^{\circ} b \cap{ }^{\circ}\left(1-a^{-} a\right)={ }^{\circ}\left(b R+\left(1-a^{-} a\right) R\right)$ then ${ }^{\circ} b \cap R a=(0) \Rightarrow b R+$ $\left(1-a^{-} a\right) R=R$. It is obvious that the latter implies $a b R=a R$. Symmetry now yields the second result.

THEOREM 2. Let $R$ be a regular ring with unity 1 and let a, $b, c$ be elements of $R$. Suppose that $I$ and $J$ are right ideals in $R$. The following are equivalent. 

(i) $R$ is finite
(ii) $a R \cong b R$ together with $a R \cong b R$ or $R a \cong R b \Longrightarrow a R=b R$
(iii) $I \subseteq b R, I \cong b R$
$\Longrightarrow I=b R$
(iv) $I \cong J, I \cong J, J$ complemented
$\Longrightarrow I=J$
(v) $I \cong R$
$\Longrightarrow I=R$
$\Longrightarrow a R=R$
(vii) $a R \oplus I \cong c R, c R \cong a R$
$\Longrightarrow I=(0)$
(viii) $a R \oplus b R \cong a R$
$\Longrightarrow b=0$
(ix) $\left[\begin{array}{ll}e & 0 \\ 0 & f\end{array}\right] \sim\left[\begin{array}{ll}e & 0 \\ 0 & 0\end{array}\right]$
$\Longrightarrow f=0$
(x) $a R+b R \cong a R$
$\Longrightarrow b=0$
(xi) $R a^{k}=R a^{k+1} \Longrightarrow a^{k} R=a^{k+1} R$ for some $k \geqq 0$.

(3.3) (vi) $\quad a R \cong R$

An analogous and equivalent result holds for left ideals.

Proof. (i) $\Rightarrow$ (ii): Let $e=a a^{+}$and $f=b b^{+}$. Then $e \sim f$ and $e R \subseteq f R$. By Theorem $1 e R=f R$ and so $a R=b R$.

(ii) $\Rightarrow$ (iii): Since $I \cong b R \Rightarrow I=a R$ for some $a \in R$, the result is clear.

(iii) $\Rightarrow$ (iv): Any complemented one-sided ideal is principal in a ring with unity.

(iv) $\Rightarrow(\mathrm{v})$ : Take $J=R$ in (iv).

(v) $\Rightarrow$ (vi): Clear with $a R=I$.

(vi) $\Rightarrow$ (i): If $a b=1$ then $R b=R \Rightarrow b R \cong R$. By (vi) $b R=R$, implying that $b$ is a unit with $a$ as inverse.

(ii) $\Rightarrow$ (vii): Let $\phi: a R \oplus I \rightarrow c R$ be the isomorphism. Then $\phi(\alpha, 0)=$ $c x$ for some $x \in R, \phi(0, I)=I_{1} \subseteq c R$ and $\phi(a R, 0)=c x R$. Hence $a R \cong$ $c x R \subseteq a R$ and so by (iii) $a R=c x R \subseteq c R \subseteq a R$. Now $I_{1}=a R \cap I_{1}=$ $c x R \cap I_{1}=\phi(a R, 0) \cap \phi(0, I)=\phi[(a R, 0) \cap(0, I)]=\phi(0,0)=(0)$. Hence $I \cong I_{1}=(0)$ and so $I=(0)$ as desired.

(vii) $\Rightarrow$ (viii): Trivial.

(viii) $\Leftrightarrow$ (ix): By (1-3) $\left[\begin{array}{ll}e & 0 \\ 0 & f\end{array}\right] \sim\left[\begin{array}{ll}e & 0 \\ 0 & 0\end{array}\right] \Leftrightarrow e R \oplus f R \cong e R$ and hence setting $e=a a^{+}$and $f=b b^{+}$yields the desired equivalence.

(viii) $\Rightarrow(x)$ : External cancellation laws always imply the corresponding internal cancellation laws since the directness of the sum $a R+b R$ implies that $a R+b R \cong a R \oplus b R$.

$(\mathrm{x}) \Rightarrow$ (i): Let $e^{2}=e$, then $e R \cong R=e R+(1-e) R$ implies by (x) that $e=1$, ensuring by Theorem 1 that $R$ is finite.

(ii) $\Rightarrow$ (xi): If $R a^{k}=R a^{k+1}$ then $a^{k} R \cong a^{k+1} R \cong a^{k} R$ and hence $a^{k} R=a^{k+1} R$ guaranteeing that $\left(a^{k}\right)^{\sharp}$ exists.

$(\mathrm{xi}) \Rightarrow$ (i): This was shown in Proposition 2. 
The left analogue follow by left-right symmetry.

COROLlaRY 7. Over a finite regular ring $a R \nRightarrow R \oplus R$, and $a R \cong a R \oplus a R$ implies $a=0$. In particular $R \nRightarrow R \oplus R$.

REMARK. In Example 2 we shall see that the isomorphic inclusion law for right ideals:

$$
I \subseteq J, I \cong J \Longrightarrow I=J,
$$

will not be true in general in a finite regular ring, if $J$ is not a principal ideal.

Proposition 6. Let $R$ be a finite regular ring. Then

(i) $a R \subseteq b a R \Rightarrow a R=b a R$ and $R a=R b a$

(iii) $R a \leqq R a b \Rightarrow R a=R a b$ and $a R=a b R$.

Proof. (i). $a R \cong b a R \Longrightarrow a=b a(b a)^{+} a \Longrightarrow R a=R b a(b a)^{+} a \subseteq R a(b a)^{+} a \leqq$ $R a$. Hence by Proposition $4, a R \cong \alpha(b a)^{+} \alpha R \cong \alpha R$ and thus again by (3.3) $a R=a(b a)^{+} a R \Rightarrow b a R=b a(b a)^{+} a R=a R$. Lastly, $R b a \cong R a \supseteqq$ $R b a$ and so by (3.3) $R b a=R a$. Part (ii) follows by symmetry. We note in passing that Proposition 5 applies and that, for example, in (i) $a R=b^{n} a R, R a=R b^{n} a$ for all $n=1,2, \cdots$.

CoRollary 8. In a finite regular ring $R$

(i) $a R \subseteq b a c R \Rightarrow a R=b a c R$ and $R a c=R b a c$.

(ii) $R a \subseteq R c a b \Rightarrow R a=R c a b$ and $c a R=c a b R$.

Proposition 7. Let $R$ be a subring of a finite regular ring $R^{*}$ and let $a \in R$. Then

$$
a R=a^{2} R \Longrightarrow R a=R \alpha^{2} .
$$

Proof. Since $a R=a^{2} R$, there exists $x \in R$, such that $a=a^{2} x$ and so $\alpha R^{*}=a^{2} R^{*}$. Now $R^{*}$ is finite regular and thus by (3.3) $R^{*} a=R^{*} a^{2}$, which implies that $a=y a^{2}$ for some $y \in R^{*}$. Hence $y a=y\left(a^{2} x\right)=$ $\left(y a^{2}\right) x=a x \in R$. Now let $b=y a=a x$, then $y b=y a x=b x \in R$ and also $b a=y a^{2}=a$. Thus $(y b) a^{2}=y a^{2}=a$ or $R a^{2}=R a$ as desired.

COROLlaRY 9. If $R$ is a subring of a finite regular ring and $a \in R$, then

$$
a^{k+1} R=a^{k} R \Longrightarrow R a^{k+1}=R a^{k}
$$

Proof. Since $a^{k+1} R=a^{k} R \Leftrightarrow a^{2 k} R=a^{k} R$, and $R=a^{k+1}=R a^{k} \Leftrightarrow R a^{2 k}=$ $R a^{k}$, we may apply Proposition 7 to $a^{k}$. 
It is clear from Corollaries 1 and 3 and the identity eae[e(eae $\left.)^{-} e\right] e a e=$ $e a e$, that if $R$ is finite regular, then so is the corner ring $e R e$, where $e^{2}=e \in R$. This should be compared with the following result by Kaplansky (unpublished), which states that $e R e$ inherits the unit regularity from a ring $R$. We add the proof for completeness. [W. Desch, private communication].

Proposition 8 (Kaplansky). Let $R$ be a unit regular ring and $e$ be an idempotent element in $R$. Then eRe is unit regular.

Proof. Let ere be an arbitrary element in $e R e$ and $u=(e r e+1-e)^{-}$ be a unit. Since $(1-e) u(1-e)=1-e, \operatorname{ereu}(1-e)=0,(1-e) u e r e=0$, $e u(1-e)=u(1-e)-(1-e)$ and $(1-e) u e=(1-e)(u-1)$, we have ere $(e(u-u(1-e) u) e)$ ere $=$ ere and $e(u-u(1-e) u) e \cdot e u^{-1} e=e=$ $e u^{-1} e \cdot e(u-u(1-e) u) e$.

Related to the corner ring is the following well-known result which generalizes a result of [15].

Proposition 9. Let $M$ be a right unital $A$-modules where $A$ is a ring with unity 1 , and let $R=\operatorname{End}_{A}(M)$. If $e^{2}=e \in R$ and $E=$ $\operatorname{End}_{A}(e M)$ then $e R e \cong E$.

It should be observed here with aid of Corollary 4, that if $u$ is a unit in a regular ring $R$ and $e=e^{2} \in R$, then eue is a unit in $e R e$ exactly when $(1-e) u^{-1}(1-e)$ is a unit in $(1-e) R(1-e)$. Indeed, from (3.2) we see that

$$
\begin{aligned}
e u e R=e R & \Longleftrightarrow u e R+(1-e) R=R \Longleftrightarrow e R+u^{-1}(1-e) R=R \\
& \Longleftrightarrow(1-e) u^{-1}(1-e) R=(1-e) R .
\end{aligned}
$$

4. Cancellation laws. We begin by defining four strong cancellation laws for internal direct sums of principal right ideals. Let $R$ be a ring with unity and let $a, b, c, d \in R$. We define,

$$
\begin{array}{ll}
C_{0}^{\text {in }}: & a R+b R=c R+d R, a R \cong c R \Longrightarrow b R \cong d R \\
C_{1}^{\text {in }}: & a R+b R \cong c R+d R, a R \cong c R \Longrightarrow b R \cong d R \\
C_{2}^{\text {in }}: & a R+b R \cong a R+d R \quad \\
C_{3}^{\text {in }}: & a R+b R \cong a R \quad \Longrightarrow b R \cong d R
\end{array}
$$

There are two common ways of weakening these laws. We may define for a fixed $g \in R$, 


$$
\begin{array}{lll}
C_{0^{\prime}}^{\mathrm{in}}(g): & a R+b R=g R=c R+d R, a R \cong c R \Longrightarrow b R \cong d R \\
C_{1^{\prime}}^{\mathrm{in}}(g): & a R+b R \cong g R \cong c R+d R, a R \cong c R=b R \cong d R \\
C_{2^{\prime}}^{\mathrm{in}}(g): & a R+b R \cong g R \cong a R+d R \quad & \Longrightarrow b R \cong d R \\
C_{3^{\prime}}^{\mathrm{in}}(g): & a R+b R \cong g R \cong a R & \Longrightarrow b R=(0),
\end{array}
$$

and

$$
\begin{array}{ll}
C_{0^{\prime \prime}}^{\mathrm{in}}(g): & a R+b R=c R+d R, a R \cong g R \cong c R \Longrightarrow b R \cong d R \\
C_{1^{\prime \prime}}^{\mathrm{in}}(g): & a R+b R \cong c R+d R, a R \cong g R \cong c R \Longrightarrow b R \cong d R \\
C_{2^{\prime \prime}}^{\mathrm{in}}(g): a R+b R \cong a R+d R, a R \cong g R \quad \Longrightarrow b R \cong d R \\
C_{3^{\prime \prime}}^{\mathrm{in}}(g): a R+b R \cong a R, \quad a R \cong g R \quad \Longrightarrow b R=(0) .
\end{array}
$$

These laws may be considered as local cancellation laws, specifying a property of element $a$, or element $g$ or be considered as a global cancellation law, (holding for all $a$ ) specifying a property of the ring $R$. The case $g=1$, of course, being of special interest. For any fixed $g$ it is clear that locally, and hence globally, $C_{3^{\prime}}^{\text {in }}$ and $C_{3^{\prime \prime}}^{\text {in }}$ coincide, while $C_{k^{\prime \prime}}^{\text {in }} \Leftarrow C_{k^{\prime \prime}}^{\text {in }} \Longrightarrow C_{k^{\prime}}^{\text {in }}$ for $k=0,1,2,3$. Moreover,

$$
C_{0}^{\text {in }} \Longleftrightarrow C_{1}^{\text {in }} \Longrightarrow C_{2}^{\text {in }} \Longrightarrow C_{3}^{\text {in }} \text {. }
$$

In fact, if $C_{0}^{\text {in }}$ holds and $\phi: a R+b R \rightarrow c R+d R$ is an isomorphism then $\phi(\alpha) R+\phi(b) R=c R+d R$, and so if $\alpha R \cong c R$ then $\phi(\alpha) R \cong c R$ ensuring that $b R \cong \phi(b) R \cong d R$. Analogous implications hold for the weaker cancellation laws.

We may similarly define the corresponding local or global cancellation laws for external direct sums of principal right ideals:

$$
\begin{aligned}
& C_{1}^{\mathrm{ex}}: a R \oplus b R \cong c R \oplus d R, a R \cong c R \Longrightarrow b R \cong d R \\
& C_{2}^{\mathrm{ex}}: a R \oplus b R \cong a R \oplus d R, \quad \Longrightarrow b R \cong d R \\
& C_{3}^{\mathrm{ex}}: a R \oplus b R \cong a R \quad \Longrightarrow b R=(0) \\
& C_{1^{\prime}}^{\mathrm{ex}}(g): a R \oplus b R \cong g R \cong c R \oplus d R, a R \cong c R \Longrightarrow b R \cong d R \\
& C_{2^{\prime}}^{\text {ex }}(g): a R \oplus b R \cong g R \cong a R \oplus d R, \quad \Longrightarrow b R \cong d R \\
& C_{3^{\prime}}^{\text {ex }}(g): a R \oplus b R \cong g R \cong a R \quad \Longrightarrow b R=(0)
\end{aligned}
$$

and

$$
\begin{aligned}
& C_{1^{\prime \prime}}^{\mathrm{ex}}(g): a R \oplus b R \cong c R \oplus d R, a R \cong g R \cong c R \Longrightarrow b R \cong d R \\
& C_{2^{\prime \prime}}^{\mathrm{ex}}(g): a R \oplus b R \cong a R \oplus d R, a R \cong g R \quad \Longrightarrow b R \cong d R \\
& C_{3^{\prime \prime}}^{\mathrm{ex}}(g): a R \oplus b R \cong a R, \quad a R \cong g R \quad \Longrightarrow b R=(0) .
\end{aligned}
$$

We note that $C_{0}^{\text {ex }}$ and $C_{0^{\prime \prime}}^{\mathrm{ey}}$ are trivial, while $C_{0^{\prime}}^{\mathrm{ex}}$ is impossible. Indeed, if $a R \oplus b R=c R \oplus d R$ then $a R=c R$ and $b R=d R$. It is easily seen that locally, and hence globally, 


$$
C_{1}^{\mathrm{ex}} \Longleftrightarrow C_{2}^{\mathrm{ex}} \Longrightarrow C_{3}^{\mathrm{ex}},
$$

with an analogous result for the weak external cancellation laws.

It is always true that the external cancellation laws imply the corresponding internal cancellation laws, but the converse may not be true in general. When $R$ is regular we may of course replace the ring elements in these laws by idempotents. By analogy to the above, we may define local and global internal and external cancellation laws for right ideals, and for right $R$-modules. For example,

$$
\begin{aligned}
& \complement_{1}^{\text {ex }}: A \oplus B \cong C \oplus D, A \cong C \quad \Longrightarrow B \cong D, \\
& \complement_{0^{\prime \prime}}^{\text {in }}(M): A+B=C+D, A \cong M \cong C=B \cong D
\end{aligned}
$$

and

$$
\int_{0^{\prime}}^{\mathrm{in}}(M): A+B=M=C+D, A \cong C \Longrightarrow B \cong D .
$$

The latter type of cancellation law was used by Ehrlich [4] and reduces to $C_{0^{\prime}}^{\text {in }}(1)$, when applied to complemented (and hence principal) ideals in $R$.

If we consider $\int_{0^{\prime \prime}}^{\mathrm{in}}(M)$ as a condition on the module $M$ then it is implied by Fuchs' substitution property for right $R$-modules, which states that the right $R$-module $M$ obeys the substitution property if

$$
A+B=C+D \text { and } A \cong M \cong C,
$$

implies that for suitable module $E$,

$$
A+B=E+B=E+D \text {. }
$$

Also, when considered as local conditions on $A=M$, it follows that

$$
\complement_{\imath}^{\mathrm{ex}} \Longleftrightarrow \mathfrak{C}_{\imath^{\prime}}^{\mathrm{ex}}(M) \quad i=1,2,3 .
$$

Before turning to our main comparison between finite and unit regular rings, let us first examine some of the interdependence between the above cancellation laws. First, it is clear that each cancellation law for modules implies the corresponding one for ideals which in turn implies the one for principal ideals.

By analogy to the above, it is easily seen that for right $R$-modules

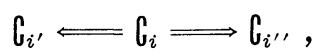

for internal as well as external cancellation laws for all possible $i$. Also,

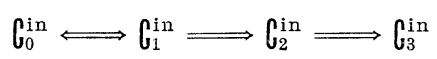

and 


$$
\mathrm{C}_{1}^{\mathrm{ex}} \Longleftarrow \mathrm{C}_{2}^{\mathrm{ex}} \Longleftarrow \mathrm{C}_{3}^{\mathrm{ex}},
$$

with analogous implications for $\complement_{i^{\prime}}$ and $\complement_{i^{\prime \prime}}$, and again $\complement_{3^{\prime}}$ and $\complement_{3^{\prime \prime}}$, being identical.

As mentioned earlier the internal laws for, say (principal) ideals, may not imply the corresponding external law. We do have however that for modules,

$$
\mathfrak{C}_{1}^{\mathrm{in}} \Longleftarrow \mathfrak{C}_{1}^{\mathrm{ex}},
$$

with analogous results for $\complement_{i^{\prime}}$ and $\complement_{z^{\prime \prime}}$. It is exactly the equivalence of the internal and external laws which appears naturally in the study of regular rings. Lastly, if we take $M=g R$ in the cancellation laws $\complement_{i^{\prime}}, i=1,2,3$, then because of the much stronger condition we may conclude that

$$
\int_{i^{\prime}}^{\mathrm{in}}(g R) \Longleftrightarrow \mathrm{G}_{i^{\prime}}^{\mathrm{ex}}(g R) \Longleftrightarrow C_{i^{\prime}}^{\mathrm{ex}}(g) \Longleftrightarrow C_{i^{\prime}}^{\mathrm{in}}(g) \quad i=1,2,3 .
$$

Indeed if, say, $i=1$ all that is needed is that $C_{1^{\prime}}^{\mathrm{in}}(g) \Rightarrow \boldsymbol{G}_{1^{\prime}}^{\mathrm{ex}}(g R)$.

Therefore, let $A \oplus B \cong g R \cong C \oplus D, A \cong C$, where $A, B, C, D$ are right $R$-modules. If $\phi: A \oplus B \rightarrow g R$ and $\psi: C \oplus D \rightarrow g R$ are the isomorphism, then there is $a \in A, b \in B$, such that $\phi(a, 0)+\phi(0, b)=$ $\phi(a, b)=g$ and hence $g R=\phi(a, 0) R+\phi(0, b) R$ with $\phi(a, 0) R \cong A$, $\phi(0, b) R \cong B$. Likewise, there are $c \in C, d \in D$ such that

$$
g R=\psi(c, 0) R+\psi(0, d) R \quad \psi(c, 0) R \cong C, \psi(0, d) R \cong D .
$$

Since $\phi(a, 0) R \cong \psi(c, 0) R$, it follows by $C_{1^{\prime}}^{\mathrm{in}}(g)$ that $C \cong \phi(0, b) R \cong$ $\psi(0, d) R \cong D$.

By analogy to the substitution property of [5] it can be shown that the cancellation law $\complement_{1}$ is inherited by internal as well as external direct sums of modules obeying these laws.

We have now arrived at the following relationship between finiteness, unit regularity and these cancellation laws.

THEOREM 3. Let $R$ be a regular ring with unity 1 , and let $e_{i}$, $f_{i}, i=1,2$ be idempotents in $R$.

( $\alpha$ ) The following are equivalent.

1. $R$ is unit regular.

2. $C_{1}^{\mathrm{ex}}$

3. $\left[\begin{array}{ll}e_{1} & 0 \\ 0 & e_{2}\end{array}\right] \sim\left[\begin{array}{cc}f_{1} & 0 \\ 0 & f_{2}\end{array}\right], e_{1} \sim f_{1} \Longrightarrow e_{2} \sim f_{2}$.

4. $\bar{C}_{1}^{\text {in }}$

5. $C_{1^{\prime}}^{\mathrm{in}}(1)$

6. $C_{0^{\prime}}^{\text {in }}(1)$

7. $C_{1^{\prime}}^{\text {ex }}(1)$ 

8. $\quad \complement_{1}^{\operatorname{ex}}(R)$
9. $\int_{1^{\prime}}^{\text {in }}(R)$.

( $\beta$ ) The following are equivalent.

1. $R$ is finite.

2. $C_{3}^{\text {ex }}$

3. $\left[\begin{array}{ll}e_{1} & 0 \\ 0 & e_{2}\end{array}\right] \sim\left[\begin{array}{ll}f_{1} & 0 \\ 0 & f_{2}\end{array}\right], e_{1} \sim f_{1} \Longrightarrow e_{2}=0$

4. $C_{3}^{\text {in }}$

5. $C_{3^{\prime}}^{\text {in }}(1)$

6. $C_{3^{\prime}}^{\mathrm{ex}}(1)$

7. $\operatorname{lex}_{3^{\prime}}(R)$

8. $\int_{3^{\prime}}^{\text {in }}(R)$.

Proof. $(\alpha) . \quad(1) \Leftrightarrow(2)$. This has been proven by Handelman [8]. Alternatively, the unit regularity of $R_{2 \times 2}$ could be used to prove this.

$(2) \Leftrightarrow(3)$. This follows from (1.3) on rewriting $C_{1}^{\text {ex }}$ using idempotents.

$(2) \Longrightarrow(4) \Longrightarrow(5) \Leftrightarrow(6)$. This always holds.

$(6) \Rightarrow(1)$. This follows from the result of Ehrlich [4] Theorem 2, which states that if $R=\operatorname{End}_{A}(M)$ is a regular ring, where $M$ is a right $A$-module, and $1 \in A$, then $R$ is unit regular exactly when

$$
M=A_{1}+H=A_{2}+K, A_{1} \cong A_{2} \Longrightarrow H \cong K .
$$

Since regular rings are faithful, this includes the cancellation law $\operatorname{Con}_{0^{\prime}}^{\text {in }}(R)$ :

$$
R=I_{1}+J_{1}=I_{2}+J_{2}, I_{1} \cong I_{2} \Longrightarrow J_{1} \cong J_{2}
$$

for complemented right ideals, which reduces to $C_{0^{\prime}}^{\text {in }}(1)$. An alternative proof is obtained from Theorem 4 and Corollary 1 of [5]. Indeed, if $R / b R \cong R / d R$ and we pick $a, c$ such that $a R+b R=R=c R+d R$, then $a R \cong c R$. Hence by $C_{0^{\prime}}^{\text {in }}(1), b R \cong d R$, which by Corollary 1 of [5] ensures that $R$ is unit regular.

The equivalence of the latter four parts was established in (4.8).

$(\beta)$ The equivalence of (1)-(4) is contained in Theorem 2, while the equivalence of (5)-(8) follows from (4.8). Lastly, if $C_{3^{\prime}}^{1 n}$ holds and $e R \cong R=e R+(1-e) R$, then $e=1$ ensuring that $R$ is finite.

REMARK. In his paper [8], Handelman actually showed that unit regularity is equivalent to the external cancellation law $\mathrm{C}_{1}^{\text {ex }}$ for finitely generated projective modules. It is an open question whether finite regularity is equivalent to $\mathrm{C}_{3}^{\mathrm{ex}}$ for modules of this type.

We close this section with a counterexample showing that the isomorphism inclusion law (3.4) as well as the external cancellation 
law $C_{1}^{e x}$ may not be valid for non-principal right ideals in a unit regular ring.

EXAMPLE 2. Let $R$ be a unit regular ring and let

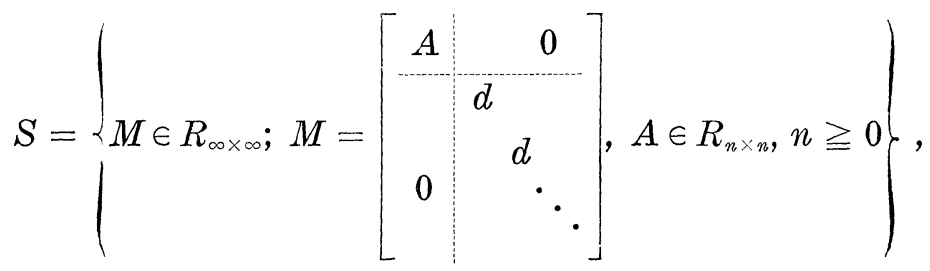

be the ring of all infinite matrices over $R$ which consist of a direct sum of a finite matrix $A$ and an infinite scalar matrix $D=\left[\begin{array}{lll}d & & 0 \\ & d & \\ & \ddots & \ddots\end{array}\right]$. It is easily verified that $S$ is again a unit regular ring. Now let $J=\left\{M \in S ; M=\left[\begin{array}{cc}A & 0 \\ 0 & 0\end{array}\right], A \in R_{n \times n}, n \geqq 0\right\}$, be the ideal of all matrices in $S$ that have zero tail and let $a=\left[\begin{array}{lll}1 & 0 & \cdots \\ 0 & 0 \\ \vdots & \ddots & \ddots\end{array}\right]$ and $I=H J$, where $H=\left[\begin{array}{c}0 \\ 1^{0} \\ 1^{\prime} \cdot \\ \cdot\end{array}\right]$ is the infinite row-shift matrix. Then $I$ and $J$ are right ideals in $S$ such that $I \subseteq J, I \cong J($ as $S$-modules) $I \neq J$ and $a R+I=$ $J, I \cong J, a R \neq(0)$.

5. Matrices over regular rings. In this section we shall give necessary and sufficient conditions for the matrix ring $R_{2 \times 2}$ to be finite regular or unit regular. We shall need some preliminary results dealing with $2 \times 2$ matrices over a regular ring $R$. If $b=p a q$, with $p$ and $q$ invertible, then the sets of reflexive inverses $\left\{b^{+}\right\},\left\{a^{+}\right\}$are related through $\left\{b^{+}\right\}=q^{-1}\left\{a^{+}\right\} p^{-1}$. Thus each reflexive inverse $b^{+}$is of the form $q^{-1} a^{+} p^{-1}$ for some $a^{+} \in\left\{\alpha^{+}\right\}$. Hence $b b^{+}=p a a^{+} p^{-1}$ for some $a^{+}$, but this is not necessarily true for $a l l a a^{+}$. We do have, however, that $b=p a q \Rightarrow b R=p a R \Rightarrow b b^{+} R=b R \cong a R=a a^{+} R \Rightarrow b b^{+} \sim a a^{+}$, for all choices of $a^{+}$and $b^{+}$. It is this "decoupling" of the idempotent $b b^{+}$from $a a^{+}$, this allows us to select suitable $b^{+}$and $a^{+}$independently from each other.

If $M=\left[\begin{array}{ll}a & c \\ b & d\end{array}\right]$ is an arbitrary matrix in the regular ring $R_{2 \times 2}$, then it can be shown that [10] there exists a reflexive inverse $M^{+}$ of $M$ such that simultaneously 


$$
M M^{+}=\left[\begin{array}{cc}
e_{1} & 0 \\
\alpha & e_{2}
\end{array}\right], M^{+} M=\left[\begin{array}{ll}
f_{1} & \beta \\
0 & f_{2}
\end{array}\right]
$$

where

$$
\begin{aligned}
e_{1} & =a a^{+}+u u^{+}\left(1-a a^{+}\right), e_{2}=s s^{+}\left(1-v v^{+}\right)+v v^{+} \\
f_{1} & =a^{+} a+\left(1-a^{+} a\right) v^{+} v, f_{2}=u^{+} u+\left(1-u^{+} u\right) s^{+} s \\
\alpha & =\left(1-s s^{+}\right)\left(1-v v^{+}\right)\left[b a^{+}+z u^{+}\left(1-a a^{+}\right)\right] \\
\beta & =\left[a^{+} c+\left(1-a^{+} a\right) v^{+} z\right]\left(1-u^{+} u\right)\left(1-s^{+} s\right)
\end{aligned}
$$

and

$$
u=\left(1-a a^{+}\right) c, v=b\left(1-a^{+} a\right), z=d-b a^{+} c
$$

with $s=\left(1-v v^{+}\right) z\left(1-u^{+} u\right)$. In these expressions $a^{+}$is arbitrary but the same choice of $a^{+}$must be used throughtout. Moreover (5.1)(5.2) remain valid if $(\cdot)^{+}$is replaced by $(\cdot)^{-}$throughout. Since $M M^{+}$ and $M^{+} M$ are triangular idempotents they are similar to diagonal matrices. Indeed, for example

$$
\left[\begin{array}{cc}
f_{1} & 0 \\
0 & f_{2}
\end{array}\right]=\left[\begin{array}{cc}
1 & f_{1} \beta-\beta f_{2} \\
0 & 1
\end{array}\right]\left[\begin{array}{ll}
f_{1} & \beta \\
0 & f_{2}
\end{array}\right]\left[\begin{array}{cc}
1 & \beta f_{2}-f_{1} \beta \\
0 & 1
\end{array}\right]
$$

We thus may state then

Proposition 10. If $M=\left[\begin{array}{ll}a & c \\ b & d\end{array}\right] \in R_{2 \times 2}$, where $R$ is a regular ring,

$$
M R_{2 \times 2} \cong\left[\begin{array}{cc}
e_{1} & 0 \\
0 & e_{2}
\end{array}\right] R_{2 \times 2}
$$

for suitable idempotents $e_{1}, e_{2}$ given by (5.2).

CoROLlaRy 10. If $E^{2}=E \in R_{2 \times 2}$, where $R$ is a regular ring, then $E \sim\left[\begin{array}{cc}e_{1} & 0 \\ 0 & e_{2}\end{array}\right]$ for suitable idempotents $e_{1}$ and $e_{2}$ in $R$.

Because (5.1)-(5.3) also hold for matrices of the form $M=$ $\left[\begin{array}{cc}A & C \\ B & D\end{array}\right] \in R_{n \times n}$, where $A \in R_{p \times p}$ and $D \in R_{q \times q}$, with $p+q=n$, we may extend these results by induction to $R_{n \times n}$. In particular, if $M=$ $\left[\begin{array}{c:c}a & \boldsymbol{c}^{T} \\ \hdashline \boldsymbol{b} & D\end{array}\right]$, where $a \in R, \boldsymbol{b}$ and $\boldsymbol{c}$ are columns in $R^{n-1}$ and $D \in R_{(n-1) \times(n-1)}$, then there exists an $M^{+}$such that $M M^{+} \approx\left[\begin{array}{cc}e_{1} & 0 \\ 0 & E_{1}\end{array}\right]$ for suitable idempotents $e_{1} \in R$ and $E_{1} \in R_{(n-1) \times(n-1)}$. Hence by Lemma $1, M R^{n} \cong$ 
$\left[\begin{array}{l}e_{1} R \\ E_{1} R^{n-1}\end{array}\right] \cong e_{1} R \oplus E_{1} R^{n-1}$, and so we may now repeat the reduction with $E_{1}$. By induction we obtain $M R^{n} \cong e_{1} R \oplus \cdots \oplus e_{n} R$ for suitable idempotents $e_{i} \in R, i=1, \cdots, n$, and thus we have

COROLlary 11. If $M \in R_{n \times n}$, where $R$ is a regular ring, then

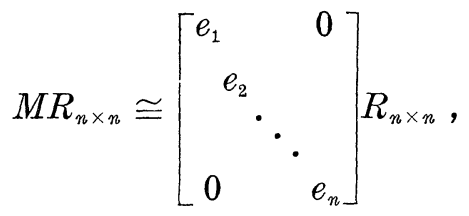

for suitable idempotents $e_{i} \in R, i=1, \cdots, n$. In particular if $E^{2}=$ $E \in R_{n \times n}$ then $E \sim\left[\begin{array}{lll}e_{1} & & 0 \\ & \ddots & \\ 0 & & e_{n}\end{array}\right]$ for some $e_{i}^{2}=e_{i} \in R, i=1, \cdots, n$.

We now recall from Theorem 2 , that if $R$ is regular then $R_{2 \times 2}$ is finite regular if and only if $M R_{2 \times 2} \cong R_{2 \times 2} \Rightarrow M R_{2 \times 2}=R_{2 \times 2}$. So if we let $E=M M^{+}$be given by (5.1), then $E R_{2 \times 2}=M R_{2 \times 2} \cong I_{2} R_{2 \times 2}$ where $I_{n}$ is the identity matrix in $R_{n \times n}$. From (1.2) we see that this holds exactly when $\left[\begin{array}{ll}e_{1} & 0 \\ 0 & e_{2}\end{array}\right] R^{2} \cong R^{2}$, or

$$
e_{1} R \oplus e_{2} R \cong R \oplus R
$$

Since $R_{2 \times 2}$ is regular exactly when $R$ is, we have proven the following.

THEOREM 4. If $R$ is a regular ring with unity 1 , then $R_{2 \times 2}$ is finite if and only if for any two idempotents $e_{1}, e_{2} \in R$,

$$
e_{1} R \oplus e_{2} R \cong R \oplus R \Longrightarrow e_{1}=e_{2}=1 \text {. }
$$

It is well-known that finiteness for $R_{2 \times 2}$ implies that of $R$. Let us now examine the converse question for regular rings. We begin by noting that

$$
\begin{aligned}
M X=I \text { for some } X \in R_{2 \times 2} & \Longleftrightarrow M M^{-}=I \text { for all } M^{-} \\
& \Longleftrightarrow M M^{+}=I \text { for some } M^{+},
\end{aligned}
$$

and hence using (5.1)-(5.2) we see that

$$
\begin{gathered}
M X=I \text { for some } X \in R_{2 \times 2} \Longleftrightarrow\left(1-u u^{+}\right)\left(1-a a^{+}\right)=0, \\
\left(1-s s^{+}\right)\left(1-v v^{+}\right)=0
\end{gathered}
$$

while

$$
\begin{gathered}
Y M=I \text { for some } Y \in R_{2 \times 2} \Longleftrightarrow\left(1-a^{+} a\right)\left(1-v^{+} v\right)=0, \\
\left(1-u^{+} u\right)\left(1-s^{+} s\right)=0 .
\end{gathered}
$$


Thus $R_{2 \times 2}$ is finite if and only if these are equivalent. We shall show now that if $R$ is regular, then $R_{2 \times 2}$ will not be finite if there exist elements $\alpha$ and $x$ in $R$, with $\alpha$ not unit regular, such that

$$
R=a R+x\left(a^{\circ}\right) .
$$

We begin by noting that (5.8) and (5.9) are, with aid of regularity, equivalent to

$$
\begin{aligned}
& \text { (i ) } \quad u R=\left(1-a a^{+}\right) R \\
& \text { (ii) } \quad s R=\left(1-v v^{+}\right) R \\
& \text { (iii) } \quad R v=R\left(1-a^{+} a\right) \\
& \text { (iv) } \quad R s=R\left(1-u^{+} u\right) .
\end{aligned}
$$

Suppose we select $c=1, d=1+b a^{+} c$ and $b=\left(1-a a^{+}\right) x$. Then $z=1$ and $u=1-a a^{+}$is idempotent so that (i) holds. Now if (5.10) holds, then by (3.2)

$$
u R=\left(1-a \alpha^{+}\right) R=\left(1-a \alpha^{+}\right) x\left(1-\alpha^{+} a\right) R=v R .
$$

Hence, by regularity $\left(1-v v^{+}\right) u=0$ so that $s=\left(1-v v^{+}\right)(1-u)=$ $1-v v^{+}$, if we pick $u^{+}=u$. It now follows that (ii) and (iv) also hold. Indeed $R s=R\left(1-v v^{+}\right)={ }^{\circ} v={ }^{\circ} u=R(1-u)$. Now consider the last identity $R v=R\left(1-a^{+} a\right)$. This implies that

$$
\left(1-a a^{+}\right) R=v R \cong\left(1-a^{+} a\right) R,
$$

and so $a a^{+} \approx a^{+} a$ since always $a a^{+} \sim a^{+} a$. But the latter is locally equivalent to $a$ being unit regular [12], which is excluded by assumption. The structure of (5.10) should be compared with the representation $R=a R+u\left(a^{\circ}\right), u$ a unit which is equivalent to $a$ being unit regular [12]. We note in passing that none of the "obvious" choices for $x$ seem to work. For example, $x=1$ implies that $a^{2} R=a R$, which if $R$ is finite, ensures that $a^{\sharp}$ exists so that $a$ is unit regular. Let us now turn to unit regular rings.

THEOREM 5. If $R$ is a regular ring with unity then $R_{2 \times 2}$ is unit regular exactly when for any idempotents $e_{1}, e_{2}, f_{1}, f_{2} \in R$,

$$
\begin{aligned}
e_{1} R \oplus e_{2} R & \cong f_{1} R \oplus f_{2} R \Longrightarrow\left(1-e_{1}\right) R \oplus\left(1-e_{2}\right) R \\
& \cong\left(1-f_{1}\right) R \oplus\left(1-f_{2}\right) R .
\end{aligned}
$$

Proof. That (5.13) is necessary is an immediate consequence of a result of Handelman (see [8, Theorem 2]).

Now suppose (5.13) holds, and that $E^{2}=E \sim F=F^{2}$. By (5.1) there exist $1-2$ inverses $E^{+}, F^{+}$such that 


$$
E E^{+} \approx\left[\begin{array}{cc}
e_{1} & 0 \\
0 & e_{2}
\end{array}\right], F F^{+} \approx\left[\begin{array}{ll}
f_{1} & 0 \\
0 & f_{2}
\end{array}\right]
$$

Hence by (1.1)-ii,

$$
I-E E^{+} \sim\left[\begin{array}{cc}
1-e_{1} & 0 \\
0 & 1-e_{2}
\end{array}\right] \text { and } I-F F^{+} \sim\left[\begin{array}{cc}
1-f_{1} & 0 \\
0 & 1-f_{2}
\end{array}\right] \text {. }
$$

But now we may apply (3.1)-ii with $E^{-}=E$ and $E==E^{+}$to show that

$$
I-E \sim I-E E^{+} \sim\left[\begin{array}{cc}
1-e_{1} & 0 \\
0 & 1-e_{2}
\end{array}\right], I-F \sim\left[\begin{array}{cc}
1-f_{1} & 0 \\
0 & 1-f_{2}
\end{array}\right] \text {. }
$$

Consequently if $E \sim F$, then

$$
E R_{2 \times 2}=E E^{+} R_{2 \times 2} \cong\left[\begin{array}{cc}
e_{1} & 0 \\
0 & e_{2}
\end{array}\right] R_{2 \times 2} \cong\left[\begin{array}{cc}
f_{1} & 0 \\
0 & f_{2}
\end{array}\right] R_{2 \times 2} \cong F R_{2 \times 2},
$$

and so by (1.3) $e_{1} R \oplus e_{2} R \cong f_{1} R \oplus f_{2} R$. Using (5.13) and (1.3) once more yields $\left[\begin{array}{cc}1-e_{1} & 0 \\ 0 & 1-e_{2}\end{array}\right] \sim\left[\begin{array}{cc}1-f_{1} & 0 \\ 0 & 1-f_{2}\end{array}\right]$ as desired. This result is easily seen to generalize to $R_{n \times n}$, with aid of Corollary 8 .

COROLlaRy 12. If $R$ is unit regular then (5.13) holds.

We may also use Theorem $3 \alpha$ to characterize the unit regularity of $R_{2 \times 2}$.

THEOREM 6. If $R$ is a regular ring then $R_{2 \times 2}$ is unit regular if and only if for any idempotents $e_{i}, f_{i}, g_{i}, h_{i} \in R, i=1,2$,

$$
\left.\begin{array}{l}
e_{1} R \oplus e_{2} R \oplus f_{1} R \oplus f_{2} R \cong g_{1} R \oplus g_{2} R \oplus h_{1} R \oplus h_{2} R \\
\begin{array}{l}
e_{1} R \oplus e_{2} R \cong g_{1} R \oplus g_{2} R \\
\Longrightarrow
\end{array} f_{1} R \oplus f_{2} R \cong h_{1} R \oplus h_{2} R .
\end{array}\right\}
$$

Proof. By Theorem $3 \alpha, R_{2 \times 2}$ is unit regular if and only if for all $A, B, C, D \in R_{2 \times 2}$ such that

$$
A R_{2 \times 2} \oplus B R_{2 \times 2} \cong C R_{2 \times 2} \oplus D R_{2 \times 2} \text { and } A R_{2 \times 2} \cong C R_{2 \times 2},
$$

it follows that $B R_{2 \times 2} \cong D R_{2 \times 2}$. Suppose now that (5.14) and (5.15) hold. Then by (5.4) there exist $e_{i}, f_{i}, g_{i}$ and $h_{i}, i=1,2$ such that

$$
\begin{aligned}
A R_{2 \times 2} \cong & {\left[\begin{array}{ll}
e_{1} & 0 \\
0 & e_{2}
\end{array}\right] R_{2 \times 2} \cong\left[\begin{array}{cc}
g_{1} & 0 \\
0 & g_{2}
\end{array}\right] R_{2 \times 2} \cong C R_{2 \times 2}, \text { and } } \\
& {\left[\begin{array}{cc}
e_{1} & 0 \\
0 & e_{2}
\end{array}\right] R_{2 \times 2} \oplus\left[\begin{array}{cc}
f_{1} & 0 \\
0 & f_{2}
\end{array}\right] R_{2 \times 2} \cong\left[\begin{array}{cc}
g_{1} & 0 \\
0 & g_{2}
\end{array}\right] R_{2 \times 2} \oplus\left[\begin{array}{cc}
h_{1} & 0 \\
0 & h_{2}
\end{array}\right] R_{2 \times 2} \cdot }
\end{aligned}
$$


With aid of Lemma 1 this implies that $e_{1} R \oplus e_{2} R \cong g_{1} R \oplus g_{2} R$ and $e_{1} R \oplus e_{2} R \oplus f_{1} R \oplus f_{2} R \cong g_{1} R \oplus g_{2} R \oplus h_{1} R \oplus h_{2} R$. Hence by (5.14) $\left[\begin{array}{cc}f_{1} & 0 \\ 0 & f_{2}\end{array}\right] R^{2} \cong\left[\begin{array}{cc}h_{1} & 0 \\ 0 & h_{2}\end{array}\right] R^{2}$ which by Lemma 1 ensures that

$$
B R_{2 \times 2} \cong\left[\begin{array}{cc}
f_{1} & 0 \\
0 & f_{2}
\end{array}\right] R_{2 \times 2} \cong\left[\begin{array}{cc}
h_{1} & 0 \\
0 & h_{2}
\end{array}\right] R_{2 \times 2} \cong D R_{2 \times 2}
$$

Conversely, if $R_{2 \times 2}$ is unit regular, it suffices to take

$$
A=\left[\begin{array}{cc}
e_{1} & 0 \\
0 & e_{2}
\end{array}\right], B=\left[\begin{array}{cc}
f_{1} & 0 \\
0 & f_{2}
\end{array}\right], C=\left[\begin{array}{cc}
g_{1} & 0 \\
0 & g_{2}
\end{array}\right] \text { and } D=\left[\begin{array}{cc}
h_{1} & 0 \\
0 & h_{2}
\end{array}\right]
$$

and apply Theorem $3 \alpha$ and Lemma 1.

REMARK. We may replace the idempotents in Theorem 6 by arbitrary regular ring elements.

Our last result will be the converse of a theorem by Henriksen [13].

THEOREM 7. If $R_{n \times n}$ is unit-regular for some $n \geqq 1$, then $R$ is unit regular.

Proof. It is well-known that if $R_{n \times n}$ is regular with identity for some $n \geqq 1$, then so is $R$. So let $e \sim f$ with $e=p \hat{p}, f=p \hat{p}$ and $p \in e R f, \hat{p} \in f R e$. Then $E=\left[\begin{array}{cc}e & 0 \\ 0 & I_{n-1}\end{array}\right]=\left[\begin{array}{cc}p & 0 \\ 0 & I_{n-1}\end{array}\right]\left[\begin{array}{cc}\hat{p} & 0 \\ 0 & I_{n-1}\end{array}\right]=P \hat{P}$ and $F=\left[\begin{array}{cc}f & 0 \\ 0 & I_{n-1}\end{array}\right]=\left[\begin{array}{cc}\hat{p} & 0 \\ 0 & I_{n-1}\end{array}\right]\left[\begin{array}{cc}p & 0 \\ 0 & I_{n-1}\end{array}\right]=\hat{P} P \quad$ where $P \in E R_{n \times n} F=$ $\left[\begin{array}{cc}e R f & ? \\ ? & ?\end{array}\right]$ and $\hat{P} \in F R_{n \times n} E=\left[\begin{array}{cc}f R e & ? \\ ? & ?\end{array}\right]$. Hence $E \sim F$ and so by the unit regularity of $R_{n \times n}, I-E \sim I-F$. That is,

$$
\left[\begin{array}{cc}
1-e & 0 \\
0 & 0
\end{array}\right] R_{n \times n} \cong\left[\begin{array}{cc}
1-f & 0 \\
0 & 0
\end{array}\right] R_{n \times n}
$$

as $R_{n \times n}$-modules, which implies by (1.2) that

$$
\left[\begin{array}{c}
(1-e) R \\
0
\end{array}\right]=\left[\begin{array}{cc}
1-e & 0 \\
0 & 0
\end{array}\right] R^{n} \cong\left[\begin{array}{cc}
1-f & 0 \\
0 & 0
\end{array}\right] R^{n}=\left[\begin{array}{c}
(1-f) R \\
0
\end{array}\right]
$$

as $R$-modules.

Hence $(1-e) \sim(1-f)$ as desired.

We remark that this result may also be obtained from Theorem 5 extended to $R_{n \times n}$, which is far less transparent however.

6. Conclusions. We have compared some of the properties of finite regular and unit regular rings, and have shown that both are 
closely related to the study of generalised inverses on the one hand, and a study of cancellation laws on the other. We have seen that the finiteness property may often replace the concept of rank (cf. Prop. 6) in matrix calculations, and that the finiteness of $R_{2 \times 2}$ depends, for a regular ring on the existence of certain nonunit regular elements.

It will be of interest to study the semigroup and subgroup structure of finite regular and unit regular rings.

We conclude with several open problems.

1. If $R$ is finite does $a^{2} R=a R \Rightarrow R a^{2}=R a$ ?

2. Does $C_{1}^{\text {in }}$ imply regularity?

3. Does $C_{1}^{\text {in }} \Rightarrow C_{1}^{\text {ex }}$ in arbitrary rings? If the answer to 2 is affirmative, then the answer is yes.

4. Is a finite regular subring of a unit regular ring also unit regular?

5. Is finite regularity equivalent to $C_{3}^{e x}$ for finitely generated projective modules?

6. When does $I \cong J, I \cong J \Rightarrow I=J$ for right ideals?

7. If $R$ is finite regular can $R \oplus R \cong R \oplus R \oplus R$ as $R$-modules hold?

8. Is the $(k, l)$ property inherited by $R_{2 \times 2}$ ?

9. What sort of finite regular ring satisfy $C_{2}^{\text {in }}$ or $C_{2}^{\text {ex }}$ ?

ACKNOWLEDGEMENT. We wish to thank the referee for several valuable suggestions.

\section{REFERENCES}

1. A Ben Israel and T. N. E. Greville, Generalized Inverses Theory and Applications, Wiley, New York, 1974.

2. M. P. Drazin, Pseudo-inverses in associated rings and semigroups, Amer. Math. Monthly, 65 (1958), 506-514.

3. G. Ehrlich, Unit regular rings, Portugal Math., 27 (1969), 209-212.

4. - Units and one-sided units in regular rings, Trans. Amer. Math. Soc., 216 (1976), 81-90.

5. L. Fuchs, On the substitution property for modules, Monatsh, Math., (1971), 198-204.

6. K. R. Goodearl, Prime ideals in regular self-injective rings, Canad. J. Math., 25 (1973), 829-839.

7. K. R. Goodearl and D. Handelman, Simple self-injective rings, Communications in Algebra, 3 (1975), 797-834.

8. D. Handelman, Perspectivity and cancellation in regular rings, J. of Algebra, to appear.

9. R. E. Hartwig, Generalized Inverses, EP Elements and Associates, to appear.

10. - Block generalized inverses, Arch. Ratl. Mech. Anal., 61 (1976), 197-251.

11. R. E. Hartwig and J. Shoaf, Group inverses and Drazin inverses of bidiagonal and triangular Toeplitz matrices, submitted for publication.

12. R. E. Hartwig and J. Luh, A note on the group structure of unit regular ring elements, submitted for publication. 
13. M. Henriksen, On a class of regular rings that are elementary divisor rings, Archiv der Mathematik, 24 (1973), 133-141.

14. I. Kaplansky, Rings of Operators, W. A. Benjamin Inc., New York, 1968.

15. I. Vidav, Modules over regular rings, Mathematica Balkanica, 1 (1971), 287-292.

Received August 17, 1976 and in revised form October 1, 1976.

North Carolina State University-Raleigh

RALEIGH, NC 27607 



\section{PACIFIC JOURNAL OF MATHEMATICS}

EDITORS

RICHARD ARENS (Managing Editor)

University of California

Los Angeles, California 90024

R. A. Beaumont

University of Washington

Seattle, Washington 98105
J. DUGUNDJI

Department of Mathematics

University of Southern Californı

Los Angeles, California 90007

D. Gilbarg and J. Milgram

Stanford University

Stanford, California 94305

\section{ASSOCIATE EDITORS}

E. F. BECKENBACH

B. H. NeumanN

F. WOLF

K. YoSHIDA

\section{SUPPORTING INSTITUTIONS}

UNIVERSITY OF BRITISH COLUMBIA

CALIFORNIA INSTITUTE OF TECHNOLOGY

UNIVERSITY OF CALIFORNIA

MONTANA STATE UNIVERSITY

UNIVERSITY OF NEVADA

NEW MEXICO STATE UNIVERSITY

OREGON STATE UNIVERSITY

UNIVERSITY OF OREGON

OSAKA UNIVERSITY
UNIVERSITY OF SOUTHERN CALIFORNIA

STANFORD UNIVERSITY

UNIVERSITY OF TOKYO

UNIVERSITY OF UTAH

WASHINGTON STATE UNIVERSITY

UNIVERSITY OF WASHINGTON

AMERICAN MATHEMATICAL SOCIETY

NAVAL WEAPONS CENTER 


\section{Pacific Journal of Mathematics}

Vol. 69 , No. 1

May, 1977

V. V. Anh and P. D. Tuan, On starlikeness and convexity of certain analytic

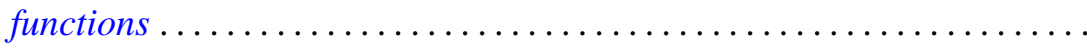

Willard Ellis Baxter and L. A. Casciotti, Rings with involution and the prime

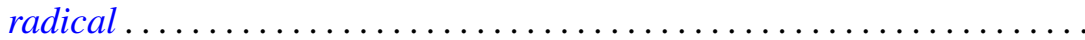

Manuel Phillip Berriozabal, Hon-Fei Lai and Dix Hayes Pettey,

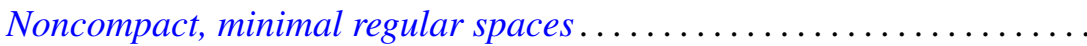

Sun Man Chang, Measures with continuous image law ................

John Benjamin Friedlander, Certain hypotheses concerning

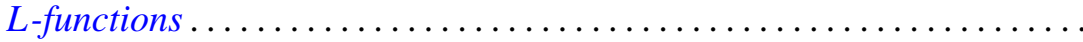

Moshe Goldberg and Ernst Gabor Straus, On characterizations and

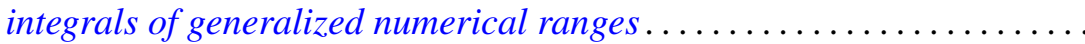

Pierre A. Grillet, On subdirectly irreducible commutative semigroups ...... 55

Robert E. Hartwig and Jiang Luh, On finite regular rings ..............

Roger Hugh Hunter, Fred Richman and Elbert A. Walker, Finite direct sums of cyclic valuated p-groups ........................... 97

Atsushi Inoue, On a class of unbounded operator algebras. III ......... 105

Wells Johnson and Kevin J. Mitchell, Symmetries for sums of the Legendre

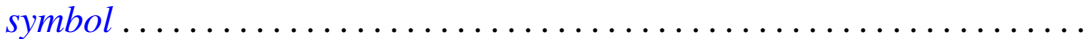

Jimmie Don Lawson, John Robie Liukkonen and Michael William Mislove,

Measure algebras of semilattices with finite breadth

Glenn Richard Luecke, A note on spectral continuity and on spectral properties of essentially $G_{1}$ operators ...............

Takahiko Nakazi, Invariant subspaces of weak-* Dirichlet algebras . .

James William Pendergrass, Calculations of the Schur group ...

Carl Pomerance, On composite $n$ for which $\varphi(n) \mid n-1$. II. . .

Marc Aristide Rieffel and Alfons Van Daele, A bounded operator approach to Tomita-Takesaki theory........................

Daniel Byron Shapiro, Spaces of similarities. IV. $(s, t)$-families ...

Leon M. Simon, Equations of mean curvature type in 2 independent variables.

Joseph Nicholas Simone, Metric components of continuous images of ordered compacta ............................

William Charles Waterhouse, Pairs of symmetric bilinear forms in

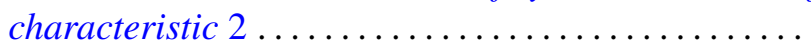

